\title{
Social, psychological and existential well-being in patients with glioma and their caregivers: a qualitative study
}

\author{
Debbie Cavers MSc PhD, Belinda Hacking MA DClinPsychol, Sara E. Erridge MD, Marilyn Kendall MA PhD, \\ Paul G. Morris MSc PhD, Scott A. Murray MD
}

\begin{abstract}
- Abstract
Background: Cerebral glioma has a devastating impact on cognitive, physical, social, psychological and spiritual well-being. We sought to understand the multidimensional experience of patients with this form of cancer as they progressed from receiving a diagnosis to the terminal phase of the disease.

Methods: We recruited patients with a suspected brain tumour from a tertiary referral centre in the United Kingdom. We interviewed patients and their caregivers at key stages of the illness: before receiving a formal diagnosis, at the start of initial treatment, after initial treatment was completed and at six months' follow-up; caregivers were also interviewed postbereavement. We interviewed the patients' general practitioners once, after treatment had been completed. We transcribed the interviews and analyzed them thematically using the constant comparative method of a grounded theory approach.
\end{abstract}

Results: We conducted in-depth interviews with 26 patients, 23 of their relatives and 19 general practitioners. We saw evidence of physical, social, psychological and existential distress even before a diagnosis was confirmed. Social decline followed a similar trajectory to that of physical decline, whereas psychological and existential distress were typically acute around diagnosis and again after initial treatment. Each patient's individual course varied according to other factors including the availability of support and individual and family resources (e.g., personal resilience and emotional support).

Interpretation: There are practical ways that clinicians can care for patients with glioma and their caregivers, starting from before a diagnosis is confirmed. Understanding the trajectories of physical, social, psychological and existential well-being for these patients allows health care professionals to predict their patients' likely needs so they can provide appropriate support and sensitive and effective communication.
Competing interests: Debbie Cavers has received payment for lectures from Queen Margaret University College. No other competing interests were declared.

This article has been peer reviewed.

Correspondence to: Dr. Scott A. Murray, scott.murray@ed.ac.uk

CMAJ 2012. DOI:10.1503 /cmaj.111622

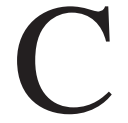
erebral glioma (hereafter glioma) is a rare but devastating cancer. ${ }^{1}$ Despite increased treatment options, average survival for the most aggressive form, glioblastoma multiforme, is less than one year. ${ }^{2}$ In addition to physical decline and increasing social isolation, ${ }^{3}$ patients may undergo a shattering of preconscious assumptions about their life and its meaning, ${ }^{4}$ causing existential anxiety. ${ }^{5-7}$ Fear around death and dying are not always verbalized, but may be expressed in other ways, such as concern for relatives or a desire to get one's affairs in order. ${ }^{8}$ Anxiety related to shock, anger, fear and uncertainty may, with time, be replaced by acceptance. ${ }^{8}$ However, little is known about how these issues interact and vary dynamically as the tumour progresses.

We sought to explore qualitatively whether patients with glioma follow archetypal trajectories of physical, social, psychological and existential well-being as their illness progresses. We investigated how people experience and deal with a diagnosis of glioma and the associated transition toward death in an effort to understand the issues patients and caregivers face and the support they need.

\section{Methods}

\section{Outcome measures}

We defined physical well-being as the absence of illness or the adaptation to symptoms such that they do not affect physical function. We defined social well-being as relating to the ability of patients and their caregivers to engage in their social network and usual lifestyle. We defined psychological well-being as it relates to emo- 
tional well-being and the ability to adjust to one's illness and its ramifications. ${ }^{9}$ We defined adjustment as the dynamic process of a person's gradual assimilation to his or her changing circumstances, drawing on a range of cognitive, emotional and social resources to manage this, incorporating psychological distress as a normal part of this process. ${ }^{5}$ We defined existential issues as relating to the meaning and purpose of life, recognizing that people may or may not use religious vocabulary to express these ideas.?

\section{Participants}

For a period of 12 months beginning in May 2006, we recruited people undergoing clinical investigations for a likely diagnosis of glioma (including glioblastoma multiforme, anaplastic astrocytoma, diffuse astrocytoma, oligodendroglioma and brain stem glioma) from a tertiary centre of clinical neurosciences in the United Kingdom. Clinicians approached potential participants by giving them an information sheet and inviting them to take part in the study before the researcher made contact.

We purposively selected patients to maximize diversity in age, sex, presenting symptoms, location and type of tumour, and to explore the widest range of potential issues. We continued sampling until we reached across-case theoretical saturation such that no new issues emerged. We sought outliers to further investigate divergent issues. We recruited patients' relatives and general practitioners through each patient.

This study was approved by the Lothian Research Ethics Committee and the Lothian Health Board.

\section{Data collection}

We used a qualitative longitudinal multiperspective technique to explore the lived experiences of patients and their caregivers. ${ }^{10,11}$ We conducted all interviews over a period of two years. Serial interviews were done with patients and their relatives up to five times over a period of 918 months: time 1, before pathological diagnosis; time 2, after diagnosis and immediately preceding treatment with chemotherapy or radiotherapy; time 3, after treatment ended; time 4 , at six months' follow-up; and time 5, postbereavement. Interviews typically lasted about 60 minutes (ranging from $30 \mathrm{~min}$ to $2 \mathrm{hr}$ ) and were usually done in the participants' own homes.

Our initial interviews with patients and relatives explored their experiences of reaching a diagnosis and adjusting to it, including existential or spiritual concerns and thoughts on death and dying. Subsequent interviews revisited important issues and discussed any changes that had occurred since the previous visit. Appendix 1 (available at www.cmaj.ca/lookup/suppl /doi:10.1503/cmaj.111622/-/DC1) provides the topic guide used by the interviewers.

We interviewed the patients' general practitioners near time 3 to gain their perspective on caring for a patient with glioma from first contact. These interviews were done either over the telephone or in person and lasted up to 30 minutes.

\section{Analysis}

We transcribed the interviews and analyzed them using the constant comparative method of a grounded theory approach. ${ }^{12}$ We used open, axial and focused coding to examine the data, progressing from "line-by-line" open coding to gradually link common codes across data to form hierarchical themes. We revised and refined our categories in an iterative comparative process. We looked for outliers, such as younger patients or unusual presentations, to test and further refine our themes. We then placed the results into a wider theoretical context (i.e., Brennan's model of adjustment emphasizing the dynamic course of illness). ${ }^{5}$

We undertook a cross-sectional analysis of all interviews at specific transitions, analyzing within and across cases serially to explore individual and group patterns. All of the interview transcripts were analyzed by Debbie Cavers; $20 \%$ of the transcripts were also read and analyzed by Scott Murray, Belinda Hacking and Paul Morris, who discussed them as a multidisciplinary group. We then developed the themes that were prevalent at various stages in the trajectories of the illness to see if typical findings or needs emerged at specific times. We did not attempt to quantitatively measure levels of distress. We used in-depth qualitative methods to explore patients' and caregivers' experiences in detail and describe dynamic patterns.

\section{Results}

We conducted 80 interviews with 26 patients (14 men, age range $21-76 \mathrm{yr}$, mean age $51 \mathrm{yr}$ ) who were undergoing investigations for glioma or who had received a confirmed diagnosis of glioma. We conducted 54 interviews with 23 of our patients' relatives, either individually or together with the patients if the patients so preferred. Only two patients declined to participate in the study.

Of the 26 patients originally identified, 21 received a confirmed diagnosis of glioma, and only these patients participated in further inter- 
views after time 1 . We also completed 20 caselinked interviews with general practitioners (see Table 1). Two participants feeling particularly distressed were referred independently by the neuro-oncology team for psychological assessment, and one caregiver made a self-referral to psychological services.

Here, we present quotations from select inter- views, ordered from diagnosis to bereavement, to provide some understanding of the trajectory followed in each of the dimensions investigated: physical, social, psychological and existential. In addition, we present quotations from interviews with general practitioners to highlight their impressions of the multidimensional distress suffered by their patients.

Table 1: Characteristics of 26 patients and their caregivers who participated in the study

\begin{tabular}{|c|c|c|c|c|c|}
\hline $\begin{array}{l}\text { Patient } \\
\text { pseudonym }\end{array}$ & $\begin{array}{l}\text { Caregiver pseudonym, } \\
\text { relationship to } \\
\text { patient }\end{array}$ & $\begin{array}{l}\text { Patient's age at } \\
\text { first interview, yr }\end{array}$ & Diagnosis & Interviews & Format of interviews \\
\hline Adam & Cindy, wife & 28 & Astrocytoma $2 / 3$ & time 2 , time $4, \mathrm{GP}$ & Separate \\
\hline Andrew & Sheila, wife & 45 & Glioblastoma multiforme & $\begin{array}{l}\text { time } 1 \text {, time } 2, \\
\text { time } 3 \text {, time } 5, \text { GP }\end{array}$ & Separate \\
\hline Angus & None & 59 & Glioblastoma multiforme & time $2, \mathrm{GP}$ & Individual \\
\hline Ann & Oliver, husband & 66 & Not a glioma & time 1 & Paired \\
\hline Barbara & John, husband & 45 & Oligodendroglioma & time 2 , time $4, \mathrm{GP}$ & Separate \\
\hline Bill & Audrey, wife & 63 & Glioblastoma multiforme & $\begin{array}{l}\text { time } 2 \text {, time } 3 \text {, } \\
\text { time } 4 \text {, time } 5, \text { PP }\end{array}$ & Paired \\
\hline Christine & None & 37 & Astrocytoma 2 & time 3, GP & Individual \\
\hline David & None & 48 & Brain stem glioma & $\begin{array}{l}\text { time } 2, \text { time } 3 \text {, } \\
\text { time } 4, G P\end{array}$ & Individual \\
\hline Deirdre & None & 56 & Glioblastoma multiforme & $\begin{array}{l}\text { time } 1, \text { time } 2, \\
\text { time } 3, \text { GP }\end{array}$ & Individual \\
\hline Ewan & Harry, father & 21 & Glioblastoma multiforme & $\begin{array}{l}\text { time 3, time 4, } \\
\text { time 5, GP }\end{array}$ & Separate \\
\hline Hannah & None & 35 & Not a glioma & time 1 & Individual \\
\hline Harriet & Alistair, husband & 64 & Glioblastoma multiforme & time 2 , time $3, \mathrm{GP}$ & Paired \\
\hline Henry & Alice, wife & 65 & Glioblastoma multiforme & $\begin{array}{l}\text { time } 1 \text {, time } 2, \\
\text { time 3, time 4, GP }\end{array}$ & Paired \\
\hline Ian & Sharon, wife & 46 & Glioblastoma multiforme & $\begin{array}{l}\text { time } 1, \text { time } 2, \\
\text { time } 3 \text {, time } 5, \text { GP }\end{array}$ & Separate \\
\hline Jenny & Bob and Diane, parents & 34 & Not a glioma & time 1 & Paired and separate \\
\hline Lois & Steven, husband & 50 & Glioblastoma multiforme & time 3, GP & Separate \\
\hline Malcolm & Joan, wife & 43 & Anaplastic astrocytoma & $\begin{array}{l}\text { time } 1 \text {, time } 2, \\
\text { time 3, GP }\end{array}$ & Paired \\
\hline Mary & $\begin{array}{l}\text { Jim, husband } \\
\text { Ailsa, daughter }\end{array}$ & 76 & Anaplastic astrocytoma & $\begin{array}{l}\text { time } 1 \text {, time } 2, \\
\text { time 5, GP }\end{array}$ & Paired \\
\hline Robert & Beth, wife & 36 & Glioblastoma multiforme & time 2, GP & Paired \\
\hline Ron & Betty, wife & 64 & Glioblastoma multiforme & $\begin{array}{l}\text { time 2, time 3, } \\
\text { time 4, GP }\end{array}$ & Paired \\
\hline Sandra & Stuart, husband & 46 & Not a glioma & time 1 & Paired \\
\hline Sandy & $\begin{array}{l}\text { Julie, wife } \\
\text { Cara, daughter }\end{array}$ & 47 & Glioblastoma multiforme & $\begin{array}{l}\text { time } 1 \text {, time 2, } \\
\text { time 5, GP }\end{array}$ & Separate \\
\hline Sarah & James, husband & 66 & Not a glioma & time 1 & Separate \\
\hline William & Shauna, wife & 64 & Glioblastoma multiforme & $\begin{array}{l}\text { time } 2 \text {, time } 3 \text {, } \\
\text { time } 4 \text {, time } 5 \text {, GP }\end{array}$ & Paired \\
\hline Wilson & Angie, wife & 58 & Glioblastoma multiforme & $\begin{array}{l}\text { time } 2, \text { time } 3 \text {, } \\
\text { time } 4 \text {, time } 5, \mathrm{GP}\end{array}$ & Separate \\
\hline Winnie & Norman, husband & 59 & Glioblastoma multiforme & $\begin{array}{l}\text { time } 1 \text {, time } 2, \\
\text { time 5, GP }\end{array}$ & Paired \\
\hline
\end{tabular}

Note: time 1 = before diagnosis, time 2 = after diagnosis and before treatment, time $3=$ after treatment, time $4=$ six months' follow-up, time $5=$ bereavement, $\mathrm{GP}=$ interview with general practitioner around time 3. 


\section{Dynamic physical trajectory}

Initial physical problems with which patients presented varied from a sudden isolated seizure with rapid diagnosis, to more gradual symptoms including headache and nausea:

The little funny turns which were almost like, it felt to me, almost if I was having the start of a seizure ... Just a bit of a shuddering in my head and wanting to just be lying down. But never ever lost consciousness or anything like that. Just, you know, I was working. I was scared, I was very scared.

- Sandra, 46-year-old woman with a suspected glioma, interview before diagnosis (time 1)

Physical and cognitive symptoms tended to increase in number and severity with time, with a substantial impact on patients' strength, mobility, communication, understanding, behaviour and appearance. Problems with memory, speech and language could become particularly distressing:

My memory is not the same, not the same as what it used to be like ... trying to read things, you just, you read a wee bit and then you get fed up with it. Concentration is not there either.

- Robert, 36-year-old man with glioblastoma multiforme, interview after diagnosis (time 2)

When you are starting a sentence, er, and you can't think of the right word and you come out with some, you tried desperately. You know and I can see you being annoyed with the situation intensely because you can't think of the right word.

- Alistair, husband of Harriet (64-year-old woman with glioblastoma multiforme), interview after treatment (time 3)

Most patients had a period of stability before their tumours gradually progressed, leading to more debilitating physical symptoms and cognitive decline. The burden of both the disease and its treatment were evident:

Sometimes I get a bit tired ... I think, the first few [treatments] I didn't really feel it. But the last couple I've felt a bit tired.

- Ewan, 21-year-old man with glioblastoma multiforme, interview during follow-up (time 4)

Again, physical symptoms increased in number and severity as death approached:

And then eventually all the seizures started coming back again. And they were just like happening all the time. There was one weekend, he had 26, it was just like unbelievable.

- Sheila, wife of Andrew (45-year-old man with glioblastoma multiforme), interview postbereavement (time 5)

\section{Dynamic social trajectory}

The lives of patients and their caregivers were substantially disrupted at the time of diagnosis. Their time was occupied with in-patient stays, visits to hospital and recovering from surgery, leaving little time to invest in their social wellbeing. However, many people gained support from family and friends visiting them. While waiting for her diagnosis (time 1), Ann (a 66year-old woman with suspected glioma) suggested "It must be terrible for somebody that haven't got er, family to support them."

Local support and information services were available, but little used. However, these services were valued by those who did use them:

So [patient] could ask questions because as I say there's things he wants to know. And she was very helpful as well and very positive as well about the whole thing. That was very helpful.

— Sheila, interview at time 2

For most people whose disease was stable, there was a strong desire to return to "normal" socially after treatment (or a "new normal," depending on abilities). For some patients, the effort of socializing posed a barrier to returning to life as normal:

We stopped doing that because it's ... likes the pubs and all that sort of stuff, just gave up that because you cannae [cannot] really get into that at all.

- Andrew, 45-year-old man with glioblastoma multiforme, interview at time 3

Caregivers found it difficult to continue to work and participate in other activities; some identified a change in gender roles:

It was at least an hour later than I really wanted to depart (for work), when I got finished. Erm, doing household jobs and what have you ... Erm, I've no doubt other things that might tire me out and wear me out, like erm, organizing the washing, drying of clothes.

— Alistair, interview at time 3

As the disease progressed, the sense of isolation increased:

I'm seeing people in a different context, but they are kind of one-off things as opposed to like a normal social life. ... 'Cause, I mean, I, the types of stuff I was doing that I mentioned earlier, the exhibitions, you know, book shops and things, I'm just not able to do any more. So, you know, gradually I suppose, I've been cut off from society that way.

— David, 48-year-old man with a brain stem glioma, interview at time 4 
For those interviewees who practised a faith, meeting church members was a valued source of social, emotional and spiritual support:

There's a great boost from all these different people plus the fact of the, the people in [town] that go to the church in our part of the community there, I mean, they're very, very supportive.

- Audrey, wife of Bill (63-year-old man with glioblastoma multiforme), interview at time 4

During the terminal phase of the illness, people's social lives dwindled alongside their physical and cognitive abilities. Often, patients became house- or bed-bound and were unable to communicate.

\section{Dynamic psychological trajectory}

Before formal diagnosis, with uncertainty at its peak, most patients felt an immediate drop in their psychological well-being. Patients' anxiety was most acute while waiting for a formal confirmation of a malignant growth.

I was scared when they told me, when they said there was a tumour. It knocked me for six. I thought, oh my God, I'm gonna die?

- Ian, 46-year-old man with glioblastoma multiforme, interview at time 1

I wanted to know, what, have I got three months, have I got six months, have I got a year? Or does anybody know?

- Sarah, 66-year-old woman with suspected glioma, interview at time 1

Caregivers reported great stress in caring for their loved ones while waiting for the diagnosis:

Cos the the pressure is really enormous, you know, enormous. But it's, I've never, I never visualized in my life, having to do anything as difficult as what's happening now you know.

\section{— James, husband of Sarah, interview at time 1}

During the initial treatment period, when patients received radiotherapy and attended regular clinics, anxiety generally lessened as patients were assured by caring clinicians:

He is such a warm person. And makes you somehow just, you can trust him. You know that you are in very good hands and he kind of radiates that presence. So I think that affects how, when we were talking about this.

- Joan, wife of Malcolm (43-year-old man with anaplastic astrocytoma), interview at time 2
Indeed, participants placed a great deal of importance on the style of communication used by clinicians:

It does help a lot, you know, it helps to make the patient feel better as well actually. You know and they can sort of communicate with somebody and have a smile.

— James, interview at time 1

During treatment, most patients strongly desired to return to normal and to make the most of their remaining time, aware that progression of their disease was likely:

I think for me, because, because I'm that good I just keep going on as normal until I really do feel no well. And then I can start changing.

— Lois, 50-year-old woman with glioblastoma multiforme, interview at time 2

Anxiety and concerns about life expectancy, future pain and loss of control at the end of life were voiced at this time:

That's what I said earlier, you know, when the pain starts, what do they do? When I start losing my faculties, what do I do? I don't know.

- Wilson, 58-year-old man with glioblastoma multiforme, interview at time 2

During the interviews at time 3 , after treatment, anxieties were often expressed by patients:

What are the things that could happen? I mean, what, is it going to be a stroke or is it going what? It might be a bit morbid to start thinking what will it be?

- Henry, 65-year-old man with glioblastoma multiforme, interview at time 3

For caregivers, dealing with progressive personality changes was most distressing:

It's made it sort of, unbearable sometimes ... It's hard to imagine being able to live the rest of my life with somebody with that kind of temperament.

- Sharon, wife of Ian (46-year-old man with glioblastoma multiforme), interview at time 3

Some caregivers showed symptoms of depression, particularly if they had a history of the disease:

I have been depressed in the past so perhaps that is why I am feeling more depressed than I would like. But it's just, I hadn't really decided what to do, you know, about getting [anti]depressants, I don't know.

— Joan, interview at time 3 
For some patients and their caregivers, the distress they felt that was related to the fear of dying eased with time:

Alice: And I think we're beginning to be, well I am, less frightened of it [dying].

Henry: Yep. I'm not, I have to say I am not frightened of it.

- Henry (65-year-old man with glioblastoma multiforme) and his wife Alice, interview at time 3

But it's now over a month since it stopped and he's just going from strength to strength and it's wonderful. This is like a sort of honeymoon period, if you like, you know, until they take the scan again.

— Angie, wife of Wilson, interview at time 3

During follow-up interviews, worries about the cancer's recurrence persisted:

I think, probably, that it's just normal and I am fine. But at the back of my mind, always the thought, now is this the cycle starting again.

- Wilson, interview at time 4

The progression of disease could cause a decline in mental well-being, but knowing the prognosis from the start meant patients had time to prepare for this eventuality:

You don't know how this deterioration ends up, how bad it might be or whatever. You know, because I don't want to, you know, become a, a complete burden on my sister.

— David, interview at time 4

The support of clinicians at this time was crucial, and a lack of such support was distressing. This was vocalized by Wilson, who said "Yes, I mean, basically I just, well, [doctor] didn't have any sort of bedside manner ... it was terrible."

Distress could increase again as thoughts about death became pressing. Three weeks before he died, Sandy (a 47-year-old man with glioblastoma multiforme) became increasingly frustrated, to the point of wanting to die, stating "I've kind of made up my mind that it's better to be going than live like this."

\section{Dynamic existential trajectory}

The unconfirmed possibility of a fatal brain tumour brought many patients to immediately question the meaning and purpose of their lives. Where a sense of meaning was absent, existential distress often occurred:

I was looking for answers and I haven't got any. I've tried to get answers to some of my questions and it hasn't come yet.

— Sarah, interview at time 1
The crisis made some patients more spiritually aware, with several turning to religion,

You can find yourself getting quite spiritual in situations like this too though. I think you're feeling a bit more desperate and very vulnerable.

- Sandra, interview at time 1

General sources of existential support were available, such as friends, family and professionals:

Oh definitely. Yeah, I mean ... I mean if I never had her I'd be, really be struggling.

— Andrew, interview at time 1

Existential distress appeared to lessen with time. Everyday life was more valued, and patients became more at peace with their situations in subsequent interviews:

Every day is a bonus. And you wake up in the morning and say well, I've got another day? ... You look at life and you think, 'Yes'.

— Ian, interview at time 2

Facing a life-limiting illness led many patients to adjust their priorities,

I'm looking here and I'm thinking what are we pushing for all of the time? Sometimes you should actually just sit back and enjoy what you've got and relax ... So I think that's my kind of motto now, like.

— Robert, interview at time 2

Just as at diagnosis, a firm faith in God provided spiritual comfort for some patients as their illness progressed:

I believe there's life after death ... so that way I'm not frightened of dying ... It calms me down. I know whatever happens, when it happens, will be the Lord's decision, not mine.

- William, 64-year-old man with glioblastoma multiforme, interview at time 2

As time passed, people accepted their situation as part of an ongoing process:

... And then you say well why not me. Somebody's, people get ill ... that's life. I'm doing better with myself with accepting it.

— Bill, interview at time 3

However, some participants described a lack of professional support in maintaining their hope. For example, Sheila, in her interview at time 3, stated "I wanted them to be more positive for us."

Gradually, patients were able to prepare for and accept the prospect of dying. Some partici- 
pants developed a greater appreciation for nature, whereas others were simply resigned to their deaths:

I get up early now and I like to look out at the day break you know and see the sun coming out over there it's good, I enjoy that. Nice and quiet and have my thoughts and think that's nice that's, life could be a lot worse, yeah. Just thinking what a wonderful world you know.

— Bill, interview at time 3

I am fairly pragmatic about ... life and death. Everyone is as important as everyone else. But ultimately we're all insignificant is really how I regard it.

— David, interview at time 4

Existential sadness and distress were sometimes expressed alongside finding meaning and peace in the journey toward death. There appeared to be a swaying between hope and despair as participants struggled to maintain positive attitudes.

\section{General practitioners' impressions of multidimensional distress in their patients} General practitioners reported varying involvement in their care for patients with glioma and had mixed views on their roles and responsibilities. Many physicians saw their role as providing information, follow-up, monitoring and symptom control, and were more involved in terminal care when more generic skills were needed. Several physicians reported a lack of skill and resources to care for the specialized and multidimensional needs of their patients. Some physicians were happy to address psychosocial needs:

Sometimes I feel like I am a psychologist and a social worker as well.

\section{— Robert's general practitioner}

It will be a very sad day when it comes to that the general practice doesn't involve that [supportive care]. ... I don't think I would be doing the job if it was just purely about dealing with medical symptoms and nothing else.

— General practitioner for Mary (76-year-old woman with anaplastic astrocytoma)

However, general practitioners did not always feel it was their role to personally address their patients' social, psychological and existential needs:

The health service cannot be everything to everybody. And the other people, or agencies or families or whatever have to do their bit to an extent. And I guess what we need to be aware of is that that is actually happening and if it's not happening then take steps to make it happen. So our role is to make sure that it happens, but it's not our role to provide it.

— Henry's general practitioner

No, I don't think we can, I don't think we should even aspire to meet their total needs. We can aspire to meet their total medical needs.

— Andrew's general practitioner

Most general practitioners felt they had a responsibility to meet the needs of both caregivers and patients:

But yes, I think it's very important to address their [caregivers'] er, concerns.

— Ewan's general practitioner

There was a strong theme among general practitioners' reports of becoming increasingly involved when patients had finished active treatment and were receiving palliative care in the community:

My involvement picked up with him when he was discharged back locally.

— Robert's general practitioner

Well obviously if it becomes terminal, you know, we'll obviously be in a position to help, you know ... we really would be becoming involved ... We would normally get the local palliative team, the care team involved.

— Wilson's general practitioner

There was concern among general practitioners about the lack of consensus as to who should coordinate care, and that some patients might be lost by the system and miss appropriate care at the end of life.

\section{Interpretation}

We have attempted to describe typical trajectories of social, psychological and existential wellbeing in patients with glioma. Following acute psychological and existential angst before and immediately after diagnosis, patients and their relatives worked hard, despite physical and cognitive decline, to foster a sense of hope and wellbeing. Psychological and existential well-being were challenged again after initial treatment and as the tumours progressed.

In primary care, some general practitioners saw their role as being minimal, whereas others coordinated follow-up, symptom control and emotional support for patients and their relatives. In addition, some general practitioners described their role as coming to the fore after 
patients completed initial treatment and returned to the community setting for care and support. Continuity of care was a priority for general practitioners.

Our qualitative data show the need for clinicians to understand patients' unique experiences, to communicate sensitively and empathically with patients and their caregivers as to what to expect, and to plan timely and appropriate interventions. Our results add a new and dynamic real-time perspective from before diagnosis. Using these data, we have summarized some practical suggestions for clinicians caring for patients with glioma in Box 1.

\section{Comparison with other studies}

As has been seen among patients with advanced lung cancer, ${ }^{13}$ the social trajectory of well-being declined alongside the physical. ${ }^{9}$ As with other chronic advancing illnesses (e.g., heart failure), ${ }^{13}$ patients in our study described a gradual withdrawal from their social lives. Caregivers became increasingly busy and isolated, unable to continue with their daily lives. In previous research, patients and caregivers have reported anxiety and distress resulting from social isolation, stigmati-

\section{Box 1: Practical suggestions for clinicians caring for patients with glioma and their caregivers}

At referral:

- acknowledge the distress of uncertainty while waiting for a diagnosis

Around diagnosis:

- offer more detailed information about the likely process of what will happen and the expected diagnosis

During initial treatment:

- provide an opportunity for patients to voice their problems or questions

- give supportive advice to caregivers and allow them to ask questions

- ensure immediate communication between the hospital and primary care facility

At follow-up:

- identify a named person (possibly a specialist nurse or family physician) who will maintain contact and offer psychological support and practical advice

- discuss what the future may hold and possible symptoms

- involve caregivers in discussions about practical help, where appropriate

Disease progression

- sensitively give patients the opportunity to plan their future care, and to consider options such as their resuscitation preferences, living wills and their preferred place of death

- involve caregivers in discussions about end-of-life care (with patients' consent)

- communicate the patient's care plan with all other services, including after-hours services

- maintain contact and support the caregiver

Bereavement

- provide an initial bereavement visit from a known and key professional

- offer further contact zation, feeling misunderstood and an inability to talk about their feelings or situation. .,14,15 $^{-15}$

Both professional and informal social support are central to adapting to illness., ${ }^{3,16}$ Patients with glioma have reported lower social support as their disease progressed and their functional abilities deteriorated. ${ }^{14}$ Recognizing that a person's desired level of social support may increase with greater need is vital to maximizing such support.

Psychological distress has been previously documented at diagnosis, recurrence and around death, but rarely before diagnosis. ${ }^{17}$ There is scope for better recognition and discussion of psychological issues among health professionals, patients and their families at this stage. ${ }^{18,19} \mathrm{Hav}$ ing these conversations early may help dispel fears, achieve better symptom control and facilitate discussions on end of life. ${ }^{20}$

Returning home after initial treatment did not appear to be associated with as much distress as has been seen in patients with lung cancer at the same stage. ${ }^{13}$ Caregivers sometimes had a different focus for their distress as they struggled to cope with the caring role and deal with changes in their loved ones' personalities; some reported low mood and feeling depressed. ${ }^{18}$

Although people worked hard to maintain a positive attitude, they struggled to do so and voiced fluctuations between hope and despair throughout the course of the illness. This state of flux is a normal part of the adjustment process. ${ }^{5}$ There may also be individual differences in acceptance and adjustment depending on such factors as internal resources, levels of support and available time. Although patients and caregivers reported feeling anxious and low in mood at times, this was a dynamic process and fitted with normal patterns of adjustment. Overall, participants showed remarkable resilience.

A life-limiting diagnosis requires a process of adaptation or assimilation. ${ }^{4}$ Preconscious assumptions about existence and mortality are questioned. ${ }^{5}$ Issues normally buried in everyday lives suddenly demand answers, having an unsettling effect..$^{21}$ This study supports the concept that a diagnosis of cancer sets a person on an existential quest, emphasizing the dynamic process fluctuating throughout the illness, rather than achieving existential adjustment as an outcome. ${ }^{6}$ Our results showing that patients developed a greater appreciation for friendships and the natural world were consistent with the re-evaluation necessary to find meaning in changing circumstances. ${ }^{22}$

Expressions of peace with oneself and pantheism were signs of positive transitions, as were moments of despair as patients struggled to maintain their positivity. ${ }^{22,23}$ Examining one's contribution and meaning in life enhances well- 
being and provides a sense of dignity and hope. . $^{24,25}$

There were mixed views as to whether "total" multidimensional care should be within the scope of practice of general practitioners caring for patients with glioma. Although some physicians felt that this should be their aim, others reported problems with providing total care related to resources, competence and communication skills. Difficulties in communicating with patients about sensitive topics have been highlighted previously. ${ }^{26}$ However, the need to provide multiple dimensions of care is evident. ${ }^{1}$ Furthermore, the need for increased research and evaluation of interventions to improve psychosocial care for people with glioma has been identified as a priority. ${ }^{27}$

\section{Limitations}

Although a variety of patients with glioma were interviewed, all of our participants were from southeast Scotland. Thus, the applicability of these findings to different national, social, ethnic and religious groups is unknown. Our study could have benefitted from including multiple sites, but we were constrained by practicalities including costs, personnel and limited time in which to complete the research.

We set out to gain insight on the experiences of patients and caregivers, in their own words. We felt that detailed discussions of physical, social, psychological and existential well-being provided the information we needed to understand these experiences. Our qualitative approach allowed an in-depth understanding of the issues that patients found most distressing. Serial, multiperspective interviews enabled us to track and compare changes in perceived problems as patients approached death, and to compare needs at various key times in this trajectory. ${ }^{10,11}$ However, although qualitative data provide rich and useful insights to the issues we studied, an added quantitative measure of distress and adjustment would have allowed us to compare our study group with the wider population.

Our lack of a formal clinical interview or tool assessing clinical depression and anxiety disorders meant we could not determine the actual incidence of these conditions in our study group. Further research could explore specific dimensions to quantify the dynamic patterns of wellbeing, although relevant tools have not yet been validated longitudinally. ${ }^{28}$

Although the serial approach has distinct advantages, ${ }^{10}$ we had some difficulty capturing the terminal phase owing to high attrition. Moreover, the different components of well-being are sometimes difficult to disentangle. Finally, $\operatorname{cog}$ nitive decline made it difficult to separate the organic symptoms of the disease from psychological distress and depression. ${ }^{29}$

\section{Conclusion}

The key to good clinical care for those who are likely to die in the relatively near future is to understand what matters to them and how they may die, and to enable appropriate planning and communication to minimize and manage their distress. Understanding the typical patterns of social, psychological and existential distress alongside physical decline enables the provision of supportive care and appropriate communication when patients and their caregivers need it most. This approach acknowledges the dynamic quality of illness and the adjustment process, showing the need for changing the support provided at key stages. Acute psychological and existential distress frequently occurs during the diagnostic process. Targeting support and open communication at this initial stage is necessary and may influence positive adaptation to the illness.

\section{References}

1. Davies E, Hopkins A. Improving care for patients with malig nant cerebral glioma, London (UK): Royal College of Physicians of London; 1997.

2. Chang SM, Parney IF, Huang W, et al. Patterns of care for adults with newly diagnosed malignant glioma. JAMA 2005;293:557-64.

3. Janda M, Eakin EG, Bailey L, et al. Supportive care needs of people with brain tumours and their carers. Support Care Cancer 2006; 14:1094-103.

4. Janoff-Bulman R. Shattered assumptions: towards a new psychology of trauma. New York (NY): The Free Press; 1992.

5. Brennan J. Adjustment to cancer - coping or personal transition? Psychooncology 2001;10:1-18.

6. Little M, Jordens MC, Paul K. et al. A major category of experience of cancer illness. Soc Sci Med 1998;47:1485-94.

7. Murray SA, Kendall M, Boyd K, et al. Exploring the spiritual needs of people dying of lung cancer or heart failure: a prospective qualitative interview study of patients and their carers. Palliat Med 2004;18:39-45.

8. Adelbratt S, Strang P. Death anxiety in brain tumour patients and their spouses. Palliat Med 2000;14:499-507.

9. Murray SA, Kendall M, Boyd K, et al. Archetypal trajectories of social, psychological and spiritual wellbeing and distress in family caregivers of patients with lung cancer: secondary analyses of serial qualitative interviews. BMJ 2010;340:c2581.

10. Murray SA, Kendall M, Carduff E, et al. Use of serial qualitative interviews to understand patients' evolving experiences and needs. BMJ 2009;339:b3702.

11. Kendall M, Murray S, Carduff E, et al. Use of multiperspective qualitative interviews to understand patients' and carers' beliefs, experiences and needs. BMJ 2009;339:b4122.

12. Charmaz K. Constructing grounded theory: a practical guide through qualitative analysis. London (UK): Sage; 2006.

13. Murray SA, Kendall M, Grant E, et al. Patterns of social, psychological, and spiritual decline toward the end of life in lung cancer and heart failure. J Pain Symptom Manage 2007;34:393402.

14. Courtens AM, Stevens FCJ, Crebolder HF, et al. Longitudinal study on quality of life and social support in cancer patients. Cancer Nurs 1996;19:162-9.

15. Jones RB, Pearson J, Cawsey AJ, et al. Effect of different forms of information produced for cancer patients on their use of information, social support and anxiety: a randomised trial. $B M J$ 2006;332:942-8.

16. Burns CM, Craft PS, Roder DM. Does emotional support influ- 
ence survival? Findings from a longitudinal study of patients with advanced cancer. Support Care Cancer 2005;13:295-302.

17. The AM, Hak T, Koeter $\mathrm{G}$ et al. Collusion in doctor-patien communication about imminent death: an ethnographic study. BMJ 2000;321:1376-81.

18. Wideheim AK, Edvardsson T, Pahlson A, et al. A family's perspective on living with a highly malignant brain tumor. Cancer Nurs 2002;25:236-44.

19. Davies E, Bannon M. Opportunities for improving the quality of care in malignant cerebral glioma. J Neurol Neurosurg Psychiatry 1999;66:693.

20. Khalili Y. Ongoing transitions: the impact of a malignant brain tumour on patient and family. Axone 2007;28:5-13.

21. Strang S, Strang P. Spiritual thoughts, coping and sense of 'coherence' in brain tumour patients and their spouses. Palliat Med 2001;15:127-34.

22. Cassell EJ. The nature of suffering and the goals of medicine. Oxford (UK): Oxford University Press; 2004.

23. Sand L, Olsson M, Strang P. Coping strategies in the presence of one's own impending death from cancer. J Pain Symptom Manage 2009;37:13-22.

24. Chochinov HM. Thinking outside the box: depression, hope, and meaning at the end of life. J Palliat Med 2003;6:973-7.

25. Chochinov HM, Hack T, Hassard T, et al. Dignity therapy: a novel psychotherapeutic intervention for patients near the end of life. J Clin Oncol 2005;23:5520-5.

26. Clayton JM, Butow PN, Arnold RM, et al. Discussing end of life issues with terminally ill cancer patients and their carers: a qualitative study. Support Care Cancer 2005a;13:589-99.

27. Catt S, Chalmers A, Fallowfield L. Psychosocial and supportivecare needs in high-grade glioma. Lancet Oncol 2008;9:884-91.

28. Ziegler L, Hill K, Neilly L, et al. Identifying psychological dis- tress at key stages of the cancer illness trajectory: a systematic review of validated self-report measures. J Pain Symptom Man age 2011;41:619-36.

29. Rooney AG, Carson A, Grant R. Depression in cerebral glioma patients: a systematic review of observational studies. J Natl Cancer Inst 2011;103:61-76.

Affiliations: From the Primary Palliative Care Research Group (Cavers, Kendall, Morris, Murray), Centre for Population Health Sciences, General Practice Section, The University of Edinburgh Medical School; and the Edinburgh Cancer Centre (Hacking, Erridge), Western General Hospital, Edinburgh, UK

Contributors: Scott A. Murray, Sara E. Erridge and Belinda Hacking conceived of the study. All of the authors designed the study and sought ethical approval. Debbie Cavers collected and analyzed the data. Debbie Cavers and Scott A. Murray drafted the manuscript. All of the authors contributed to revising and editing the manuscript and approved the final version submitted for publication. Scott A. Murray is the study's guarantor.

Funding: The study was funded by a donation from a bereaved relative to the University of Edinburgh.

Acknowledgements: The authors would like to thank the participants in the study and all those who helped with recruitment, as well as Ms. Shanne McNamara and Mrs. Eilidh Wiseman for their advice and support. 